\title{
Mapping European canker spatial pattern and disease progression in apples using GIS, Tasman, New Zealand
}

\author{
Diletta Di Iorio ${ }^{1}$, Monika Walter ${ }^{2}$, Egbert Lantinga ${ }^{1}$, Huub Kerckhoffs ${ }^{3}$ and Rebecca E. \\ Campbell ${ }^{*}$ \\ ${ }^{1}$ Wageningen University and Research, Droevendaalsesteeg 4, Wageningen 6708 PB, The \\ Netherlands \\ ${ }^{2}$ The New Zealand Institute for Plant and Food Research Limited, Old Mill Rd, RD3, Motueka \\ 7198, New Zealand \\ ${ }^{3}$ Massey University, Tennent Dr, Palmerston North 4474, New Zealand \\ ${ }^{*}$ Corresponding author: rebecca.campbell@plantandfood.co.nz
}

\begin{abstract}
European canker (EC), caused by Neonectria ditissima, is an important disease in apple-producing regions in New Zealand. In order to improve plant protection, Geographic Information Systems (GIS) can be used to map plant disease location and severity in agricultural settings. Data were compiled from apple growers in Tasman, New Zealand, to investigate EC distribution over 4 years, for the period 2015-2018. ArcGIS software, including the Spatial Analyst, Interpolation and Geospatial statistics toolboxes, was used to map EC incidence at the spatial scale of orchard blocks, which allowed the identification of disease hot-spots. A clustered spatial pattern of disease was detected every year and areas with higher risk of EC were identified within the region. The spatial patterns detected were related to disease pressure over time for different apple cultivars. The use of GIS provides a platform for analysing and visually communicating disease patterns over time. Investigating disease spatial pattern allows the inference of spatial processes and further hypothesis generation to understand the pathogen.
\end{abstract}

Keywords apple, disease progression, European canker, Geographical Information Systems, GIS, hot-spots, Neonectria ditissima, spatial, temporal.

\section{INTRODUCTION}

European canker (EC), caused by Neonectria ditissima, is an important disease in appleproducing regions that have moderate temperatures and high rainfall throughout the year, e.g. Europe, the United Kingdom, Chile, and New Zealand. It has been recorded that the death rate caused by this fungal pathogen can be around $10 \%$ in the first few years of tree establishment in an orchard (Lovelidge 1995; Berrie et al. 2000). The apple varieties most susceptible to N. ditissima are the 'delicious' cultivars, 'Golden', 'Red', 'Kanzi' and 'Royal Gala'. 'Braeburn' is moderately susceptible while 'Granny Smith' is quite resistant (McCracken et al. 2003).
The main centres of apple production in New Zealand are Hawke's Bay in the North Island, and Tasman and Nelson in the South Island. All these regions are susceptible to EC, particularly Tasman, due to suitable climatic conditions (Macara 2016), variable management practices and large apple growing areas. Autumn is the most sensitive period for infections because of the high numbers of wounds caused by picking scars and leaf scars (Walter et al. 2016), coinciding with regular rainfall facilitating inoculum production. In addition, fungicides do not have label claims that allow their use during picking, leaving picking scars vulnerable to infection (Walter et al. 2017). In order to control the disease, apple 
growers generally apply between 2-6 fungicide applications during autumn after the harvest, using a range of products including copperbased formulations and captan. Further, as part of disease control in commercial orchards, visible cankers are removed by a specialist pruning crew, often about four times per year.

The pathogen, $N$. ditissima, produces two spore types: conidia (asexual spores) and ascospores (sexual spores). In New Zealand, both types of spore are available all year around whenever rainfall occurs (Walter et al. 2017). In particular, the climate in the Tasman region facilitates EC infection any time during the year as it has more regular rainfall and moderate temperatures than other apple-growing areas. Warm and wet conditions facilitate spore production and distribution, as well as lesion development. Spores are spread primarily through rain splash and secondly via wind. These dispersal mechanisms lead to non-random spatial patterns and progression of the disease (Campbell et al. 2016). In this context, a Geographical Information System (GIS) represents a powerful management tool because it enables the spatial pattern of the disease in the orchard to be investigated spatially and temporally. Such information can assist in the understanding of infection processes as well as the prediction (and therefore the control) of further European canker spread. This knowledge can then be applied to avoid unnecessary costs of inappropriate management practices, for example by developing optimal monitoring strategies (Blumenthal et al. 2001; Campbell et al. 2016, Alizadeh et al. 2017; Walter et al. 2017). Disease clustering can happen for many reasons such as the mechanism of disease spread, the occurrence of disease vectors in a certain location and the clustering of risk factors (Pfeiffer et al. 2008). Investigating patterns of disease clustering and spatial autocorrelation (non-random spatial distribution) helps to infer and understand biological and management processes, and generate testable hypotheses. Spatial analyses methods such as cluster analyses, understanding spatial autocorrelation and kernel smoothing are often used in crime detection, ecology, animal health or in public health to make predictions of risk areas and describe phenomena patterns across continuous space (Pfeiffer et al. 2008; Leitner et al. 2009; Kieran \& Martin 2013; Campos \& Fedigan 2014). These methods (traditionally used in human geography) can be extended successfully to plant health and crop disease (Nelson et al. 1999; Quinn et al. 2011).

The aim of this study was to use GIS to help understand the spatial pattern of $N$. ditissima infection in apple orchards. We investigated whether spatial clustering of the disease occurred and how the spatial pattern of disease changed over time (from 2015 to 2018) at the spatial scale of multiple orchard blocks of different apple cultivars.

\section{METHODS}

\section{Data acquisition}

Data on the incidence of EC at a tree-resolution scale in Tasman orchard blocks had been collected by growers over the previous 4 years (2015-2018). For most blocks and years, these data were collected by specialist 'canker crew' pruning teams four times per year: during preharvest; postharvest; spring; and winter. Canker incidence was recorded for each row and each year while the number of blocks included in the investigation differed year to year. Additional blocks were newly planted or included in the monitoring over time resulting in 49 blocks sampled in 2015, 60 blocks in 2016 and 2017, and 65 blocks in 2018. The canker data included trees that had one or more of the following: branch canker (trees with at least one branch infected); poled ( $50 \%$ and more of branches removed from a tree because of EC); trunk canker (trees with EC infection visible on the trunk only); removed trees (due to EC infection); and replanted trees. Branch, poled and trunk cankers were recorded as 'strikes' in a tree, removals and replants can be rare and not used in the management of every block. A 'strike' was defined as a canker from any type of wound, spur, leaf scars and thinning.

The following formula was used to compile and calculate the percentage of canker incidence from the row and seasonal data into an average 
for every block per year:

$\%$ Canker incidence $($ block scale $)=$

(Removal trees + Strike trees)

number of seasons

Total number of trees - Removal trees of the previous year

Incidence is calculated relative to the total number of trees at the block scale so the different number of blocks included each year did not bias the EC analyses presented in this paper; however, the age of the trees would be an important factor to consider in future analyses. A number of different commercial apple varieties were present in the blocks and all were similarly susceptible to EC (Bus et al. 2019; Table 1). Varieties have been numbered to anonymise their identity for display in this paper, as conclusive formal covariate analyses have not yet been published.

\section{Spatial analyses}

In order to investigate the disease spatial pattern, compiled data for each orchard block were imported in ArcGIS (v.10.6.1) using georeferenced data. From geographical coordinates, aerial photographs and grower discussion, polygons were drawn in ArcGIS to represent each orchard block to which the attributes of each block were assigned (e.g. EC incidence, variety). Orchard blocks were the spatial unit for all spatial analyses presented; therefore, the condition 'contiguity edges and corners' was specified in the software as the spatial relationship (which means all neighbouring blocks on edges and corners were considered in the spatial relationship) and Euclidean distance was chosen for global and local autocorrelation analyses. The Geographic Coordinate System used was GCS_WGS_1984 Web Mercator.

\section{Interpolation}

Kernel smoothing is a statistical interpolation method to estimate a local value as a function of neighbouring observed data. This process enables the production of a smooth surface that defines the level of potential risk areas of the actual and interpolated values across an area (Bithell 1990) and can be used to visualise the disease pattern at a multi-block spatial scale. Therefore, interpolation was performed using a kernel smoothing tool that uses local polynomial interpolation, i.e. fitting an Order-1 polynomial function using points within the defined neighbourhood (Environmental Systems Research Institute (ESRI) 2019a). The bandwidth used for the kernel function was the extent of the study area. This interpolation was carried out separately for each year of data.

Table 1 Frequency of blocks of different apple varieties grown in the study region. Apple varieties have been numbered to anonymise their identity.

\begin{tabular}{lcccc}
\hline Variety & \multicolumn{4}{c}{ Number of blocks } \\
\hline 1 & 2015 & 2016 & 2017 & 2018 \\
2 & 32 & 37 & 37 & 37 \\
3 & 8 & 12 & 12 & 12 \\
4 & 9 & 11 & 11 & 11 \\
$3+4$ (half block each) & 0 & 0 & 0 & 3 \\
5 & 0 & 0 & 0 & 1 \\
TOTAL & 0 & 0 & 0 & 65 \\
\hline
\end{tabular}


Spatial autocorrelation (Global Moran's Index and Anselin Local Moran's Index)

Spatial clustering is a term used to describe the spatial aggregation of an event pattern. Clustering of a disease in space and time can be evaluated using two main types of cluster analysis techniques, global and local clustering methods (Pfeiffer et al. 2008). Both types were used here. Firstly, the Global Moran's Index was calculated using the Spatial Autocorrelation tool in ArcGIS. The Global Moran's Index distinguishes between positively auto-correlated patterns (high values tend to be surrounded by high values, low values by low values), random patterns (neighbouring values independent of each other) and dispersed patterns (high surrounded by low, and vice versa) (Longley et al. 2005). The global index performs best with normally distributed values across the study area; therefore, histograms were checked to ensure this assumption was met (data not shown). Secondly, in order to identify where the spatial clusters of features with high or low values and the outliers were across the orchard, the Anselin Local Moran's index was run (Clusters and Outliers analysis), calculating a $\mathrm{z}$-score, a pseudo P-value (499 permutations), a colour and a code representing the cluster type for each statistically significant feature (ESRI 2019b). A high-high cluster is defined as high values surrounded by other high values and, conversely, a low-low cluster is where low values are surrounded by low values. High-low and low-high outliers occur where the spatial pattern is dispersed and neighbouring blocks are more dissimilar than expected by chance. A low-high classification indicates where low values are surrounded by significantly higher values, and high-low the opposite.

\section{Temporal changes and apple cultivar}

Each year of data was analysed independently in this study but temporal changes in the clustering and 'hot-spots' of disease were visually assessed over the 4 years of the study and also visually compared to the locations of the different apple cultivars grown. Further formal analyses of these comparisons are needed for causative factors of these relationships.

\section{RESULTS AND DISCUSSION}

Incidence of EC in trees at the whole-orchard scale showed an overall decrease over the 4 years considered independent of the number of blocks and a result of decreases in incidence in many individual blocks, and the addition of young plantings (Table 2). This result is in agreement with previous and current EC research in the Tasman region due to the widespread uptake of best management practices (Campbell et al. 2016, Walter et al. 2017). The kernel smoothing interpolation shows where the spatial distribution of EC incidence can be seen to change in intensity and location across the orchard over the 4 years considered (Fig. 1). It is difficult to quantify the change over time with an interpolation, which highlights the importance of objective statistical

Table 2 Global Moran's Index statistics for all orchard blocks for each year indicating the level of spatial autocorrelation (z-scores indicate the number of standard deviations from the mean, the higher the $\mathrm{z}$-score the more clustered the spatial pattern, negative z-scores indicate high dispersion, close to zero indicates random spatial pattern) of European canker (EC; caused by Neonectria ditissima) tree incidence.

\begin{tabular}{lllcc}
\hline Year & $\begin{array}{l}\text { Number of } \\
\text { blocks }\end{array}$ & $\begin{array}{l}\text { Total Orchard } \\
\text { EC Incidence (\%) }\end{array}$ & Z-score & Pseudo-P-value \\
\hline 2015 & 49 & 19 & 1.85 & 0.10 \\
2016 & 60 & 17 & 3.77 & 0.01 \\
2017 & 60 & 12 & 2.29 & 0.05 \\
2018 & 65 & 10 & 4.25 & 0.01 \\
\hline
\end{tabular}




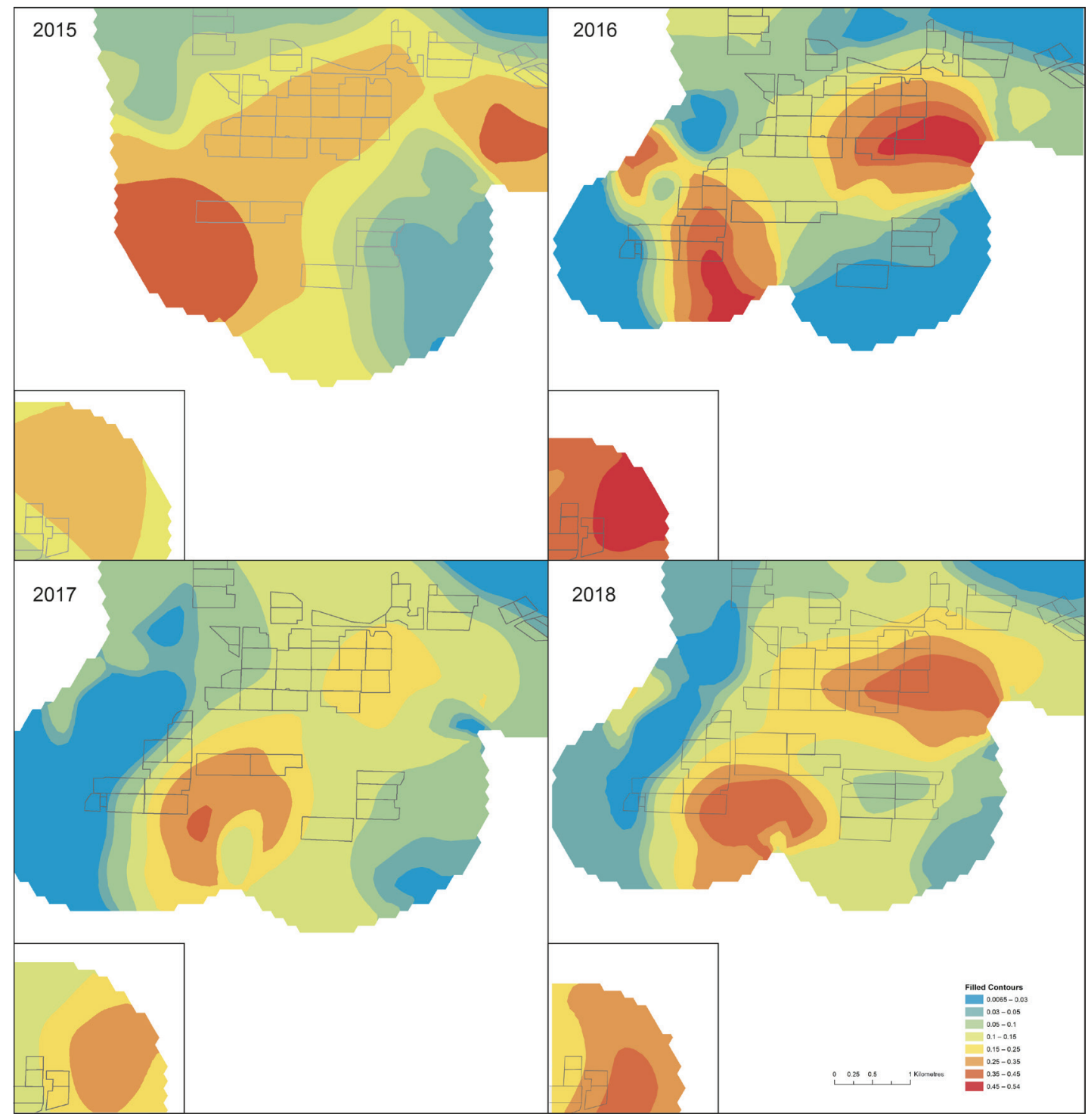

Figure 1 Spatial interpolation using kernel smoothing (polynomial order 1) for all orchard blocks over 4 years (2015-2018) performed using ArcGIS. The extent and spatial scale are the same for each year; however, the number of blocks included differs year to year and the block average tree incidence of European canker (caused by Neonectria ditissima) differs year to year (see Table 2). The inset indicates blocks belonging to the same orchard that are geographically separated from the main blocks.

analyses for detecting clustering and hotspots. It is still useful to visualise the broader-scale disease pattern across the orchard extending between the orchard blocks in order to be able to relate potential causative factors at this interpolated spatial scale. While the overall canker incidence differed from one year to another, the increases did tend to be confined to two identified areas of the orchard, the centre east and the south west (Fig. 1). 
Visual communications produced from this scale of data using GIS can be effective in quickly and simply portraying information to a wide range of audiences from industry, management, pruning teams and scientists. The spatial patterns observed also serve to generate hypotheses that can be used to inform future research about why and how these spatial patterns arise, and whether and why they could change over time. However, several factors have to be carefully considered in running geostatistical analysis and in the interpretation of results and images. The type of data, the choice of cluster detection techniques, the sampled unit size and edge effects of the study area all influence the analysis. For example, there can be statistical bias related to subjective block boundary choice (e.g. Modifiable Areal Unit Problem (MAUP); Openshaw 1984). Furthermore, different clustering algorithms can use different settings which can change the finer detail of where clusters are located. In addition, data at the block scale includes no information about disease distribution within a block, which can be highly spatially structured (data not shown). Therefore, the intended use by the growers needs to be considered to provide management maps at the correct accuracy, precision and spatial scale.

The extent of spatial autocorrelation and clustering of high disease incidence was investigated further because the Global Moran's Index indicated a significant spatially clustered distribution of canker incidence in the apple orchard for each of the 4 years (Table 2). Nonrandom spatial patterns can infer processes influencing the management of the disease. For example, the nature and mechanism of disease spread, such as rain splash dispersal, can result in different spatial patterns than wind-borne, soilborne, insect-vectored or human-transported diseases. There was slightly less statistical significance of the clustering in the first year of data collection (2015), where higher disease incidence more uniformly spread (Table 2). It is not uncommon to find that spatial patterns of disease incidence are less prominent when incidence is high (Campbell unpublished data), because the more trees with canker, the more widespread and uniform the disease becomes. If disease severity data were available, it is likely that a spatial pattern would also be prevalent with the added detail of severity, relative to basic tree incidence.

The Global Moran's Index is used to indicate whether there is significant spatial autocorrelation at the orchard scale but the Anselin Local Moran's Index is used to indicate where these hot-spots actually are within the orchard, as illustrated in Figure 2. In each of the 4 years of this study, there were always some significant high canker areas (high-high clusters) and some significantly lower canker areas (low-low-clusters). Some of these significant clusters reoccurred over multiple years, either over consecutive years or with a lag period (Fig. 2).

These spatial patterns are related to many factors and a full analysis is beyond the scope of this paper. Some explanations for the existence and subsequent changes in non-random spatial patterns are as simple as tree removal and new block planting; other explanations involve multiple environmental (e.g. rainfall, humidity), management (e.g. fungicide, pruning) and apple variety factors including the interactions between these (e.g. timing of pruning and inoculum availability).

The low-high outliers are of interest because they indicate that local disease incidence was comparatively low, despite disease pressure from neighbouring blocks. In contrast, high-low outliers indicated high localised disease incidence despite neighbouring blocks having lower incidence. The presence of these outliers could indicate a strong between-block disconnect such as shelter belts, soil characteristics or orchard management practices at the block scale.

One factor common to the hot-spots was the apple variety planted in these areas. Comparing the disease hotspots (Fig. 2) to the varieties (Fig. 3), it was observed that Variety 1 was present in all the high canker incidence clusters (Figs. $2 \& 3$ ) but not all blocks of Variety 1 occurred within clusters of high canker incidence (Figs. $2 \& 3$ ). This may be due to chance, because Variety 1 is 


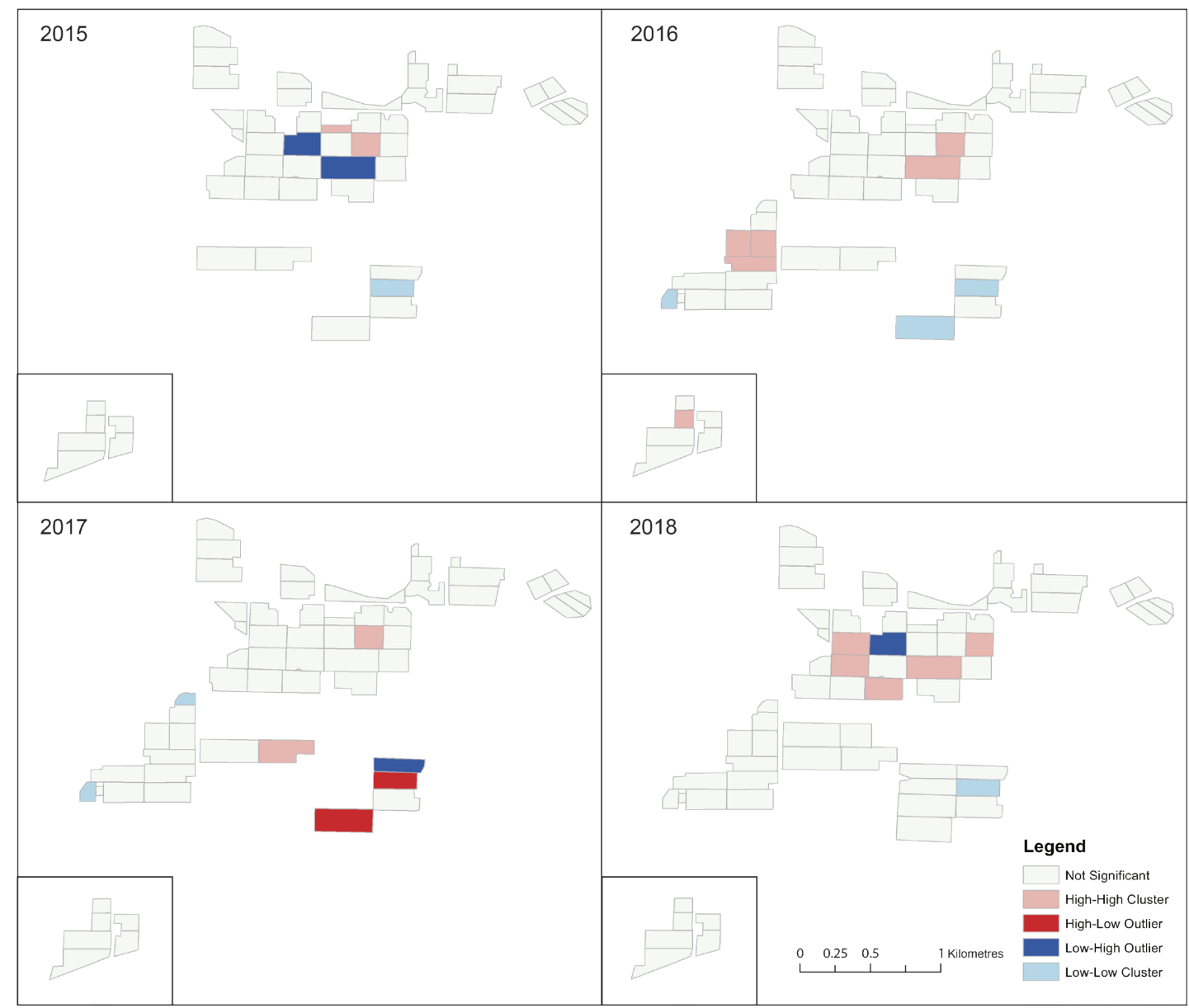

Figure 2 Locations of significant local clustering of block level European canker disease incidence over 4 years (2015-2018), using Anselin Local Moran's Index statistics. The inset indicates blocks belonging to the same orchard that are geographically separated from the main blocks. The number of blocks included differs year to year: 49 in 2015, 60 in 2016 and 2017 and 65 in 2018. High-high cluster is where there are high values surrounded by high values, and, conversely, low-low cluster is where low values are surrounded by low values. High-low and low-high outliers are where the spatial pattern is dispersed and neighbour blocks are more dissimilar than expected by chance. Low-high indicates where low values are surrounded by significantly higher values, and high-low the opposite. Varieties included in these hotspots can be compared in Figure 3.

the most widespread variety, or it could indicate subtle differences in variety susceptibility or underlying spatial factors such as soil moisture. This finding leads to many questions around whether there is an optimal planting pattern of different varieties that could decrease the risk of large, stable EC hot-spots within the orchard. For example, is disease increased by planting large monocultures of single varieties or decreased by planting multiple varieties interspersed with each other? Further research on spatial patterns of disease and their relationship with environmental factors and management practices at multiple scales is needed to better understand disease 


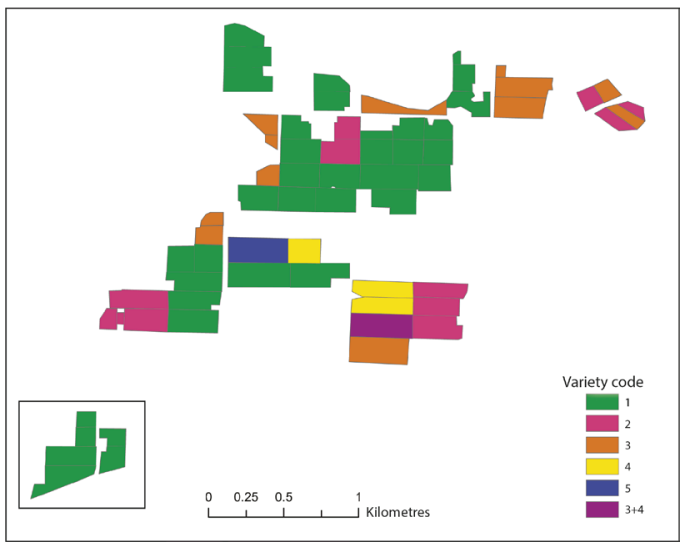

Figure 3 Spatial location of the apple varieties for the total 65 blocks in the orchard study area. Each variety has been assigned a code number to anonymise its identity. The inset indicates blocks belonging to the same orchard that are geographically separated from the main blocks.

progression. Higher resolution, row- and treescale disease data could help identify the processes behind hot-spots and their stability over time.

\section{CONCLUSION}

This research shows how GIS is a helpful tool for understanding spatial patterns of disease in horticulture. Visual displays and robust analyses of spatial data can help predict and identify disease risk areas (while avoiding map subjectivity) and also provide insights to improve management practices.

Overall, a general decline in total canker incidence occurred over time at the orchard scale, with a non-random spatial pattern indicating underlying spatial processes across the landscape. Some disease hot-spots at the block scale re-occurred each year, while others varied spatially over the four years. Understanding these spatial processes and their causes requires further robust analysis that includes environmental and management variables at multiple spatial scales.

Spatial analyses help disentangle factors that could otherwise seem random. In the application used here, it provided a greater spatial and temporal understanding of orchards, their pathosystem and their management. Therefore, GIS in horticulture can help identify and predict disease risk areas and is a powerful tool to facilitate more sustainable management practices in agriculture. However, derived maps need to be created and interpreted at the correct scale and with a sound understanding of the statistical methods and their associated limitations, to enable appropriate management decisions to be made.

\section{ACKNOWLEDGEMENTS}

We are grateful for the contribution and data sharing from the apple growers. This research was funded by The New Zealand Institute for Plant and Food Research Limited, Strategic Science Investment Fund (SSiF) and the Ministry for Primary Industries via the Sustainable Farming Fund (SFF) with New Zealand Apples \& Pears Inc. (NZAPI).

\section{REFERENCES}

Alizadeh MM, Moharrami M, Rasouli AA 2017. Geographic Information System (GIS) as a tool in epidemiological assessment of wet wood disease on elm trees in Tabriz City, Iran. Cercetari Agronomice în Moldova 2(170): 91-100.

Berrie A, Barbara D, Locke T, McCracken A 2000. Using molecular biology to study the epidemiology of canker in apple orchards. The Apple and Pear Research Council (APRC) News 24: 7-8.

Bithell JF 1990. An application of density estimation to geographical epidemiology. Statistics in Medicine 9: 691-701.

Blumenthal UJ, Fleisher JM, Esrey SA, Peasey A 2001. Epidemiology - a tool for the assessment of risk. In: Fewtrell L, Bartram J (eds). Water Quality: Guidelines, Standards \& Health Assessment of Risk and Risk Management for Water-Related Infectious Disease. IWA Publishing, London. Pp. 135-160.

Bus VGM, Scheper RWA, Walter M, Campbell RE, Kitson B, Turner L, Fisher BM, Johnston SL, Wu C, Deng CH, Singla G, Bowatte D, Jesson L, Hedderly DI, Volz RK, Chagné D, 
Gardiner SE 2019. Tree Genetics \& Genomes 15 , e25.

Campbell RE, Roy S, Curnow T, Walter M 2016. Monitoring methods and spatial patterns of European canker disease in commercial orchards. New Zealand Plant Protection 69: 213-220.

Campos AF, Fedigan LM 2014. Spatial ecology of perceived predation risk and vigilance behavior in white-faced capuchins. Behavioral Ecology 25(3): 477-486.

Environmental Systems Research Institute (ESRI) 2019a. ArcGIS Desktop Help: Environmental Systems Research Institute, Redlands, California. ArcMap latest extension, Geostatistical analyst, How Kernel interpolation with barriers works. Environmental Systems Research Institute, Redlands, California, 1995. http://desktop. arcgis.com/en/arcmap/latest/extensions/ geostatistical-analyst/how-kernelinterpolation-with-barriers-works (accessed 18 March 2019).

Environmental Systems Research Institute (ESRI) 2019b. ArcGIS Desktop Help: Spatial Statistics toolbox, Mapping Cluster toolset, How Cluster and Outlier Analysis (Anselin Local Moran's I) works. Environmental Systems Research Institute, Redlands, California 1995. http://desktop.arcgis.com/ en/arcmap/10.3/tools/spatial-statisticstoolbox/h-how-cluster-and-outlier-analysisanselin-local-m.htm (accessed 18 March 2019).

Kieran M, Martin R 2013. Using kernel methods to visualise crime data, Submission IAOS Prize for Young Statisticians. https://www.iaos-isi. org/pdf/YSP/2013-1st-Martin,Ralphs.pdf (accessed 24 April 2019)

Leitner M, Arden WB, Heukelbach J 2009. Using Kernel density interpolation to visualize the effects of mass treatment with ivermectin on Helminth prevalence in rural North East Brazil. Presented at the 24th International Cartographic Conference, Santiago de Chile, Chile, November 2009.

Longley AP, Goodchild MF, Maguire DJ, Rhind
DW 2005. Geographic Information Systems and Science. J. Wiley \& Sons, Chichester, UK. Lovelidge B 1995. Solving the apple canker mystery. Grower 123: 23-25.

Macara GR 2016. The climate and weather of Nelson and Tasman. NIWA Science and Technology Series 71. NIWA, Wellington, New Zealand. 40 p. https://www.niwa.co.nz/ node/110338 (accessed 24 April 2019).

McCracken AR, Berrie A, Barbara DJ, Locke T, Cooke LR, Phelps K, Swinburne TR, Brown AE, Ellerker B, Langrell SRH 2003. Relative significance of nursery infections and orchard inoculum in the development and spread of apple canker (Nectria galligena) in young orchards. Plant Pathology 52: 553-566.

Openshaw S 1984. The Modifiable Areal Unit Problem - Concepts and Techniques in Modern Geography. Norwich: Geo Books.

Nelson MR, Orum TV, Jaime-Garcia R, Nadeem A 1999. Application of geographic information systems and geostatistics in plant disease epidemiology and management. Plant Disease 83: 308-319.

Pfeiffer DU, Robinson TP, Stevenson M, Stevens KB, Rogers DJ, Clements ACA 2008. Spatial clustering of disease and global estimates of spatial clustering. Chapter 6. In: Spatial Analysis in Epidemiology (1st ed.). Oxford University Press: New York, NY, USA.

Quinn JA, Leyton-Brown K, Mwebaze E 2011. Modeling and Monitoring Crop Disease in Developing Countries. Proceedings of the Twenty-Fifth AAAI Conference on Artificial Intelligence: 1390-1395.

Walter M, Campbell RE, Amponsah NT, Scheper RWA, Butler RC 2017. Evaluation of biological and agrichemical products for control of Neonectria ditissima conidia production. New Zealand Plant Protection 70: 87-96.

Walter M, Roy S, Fisher BM, Mackle L, Amponsah $\mathrm{N}$, Curnow T, Campbell R, Braun P, Reineke A, Scheper RWA 2016. How many conidia are required for wound infection of apple plants by Neonectria ditissima. New Zealand Plant Protection 69: 238-245. 